\title{
Efficacy of Nucleic Acid Amplification Test (NAT) over Enzyme Linked Immuno Sorbent Assay (ELISA) in Detecting Transfusion Transmissible Infections (TTI) among Blood Donors at a Tertiary Care Centre A Comparative Study
}

\author{
Shakuntala Sunil Aramani ${ }^{1}$, Basavaraj Prabhappa Bommanahalli ${ }^{1 *}$ and Sourabh Mouneshwar Kammar ${ }^{2}$ \\ ${ }^{1}$ Department of Pathology, Gadag Institute of Medical Sciences, Karnataka, India \\ ${ }^{2}$ Medical Student, Gadag Institute of Medical Sciences, Karnataka, India
}

\begin{abstract}
Background: Background: Blood safety in India becomes prime importance with a large number of blood donors. The donated blood is screened for transfusion transmissible infections (TTI) using immunoassays and nucleic acid amplification Test (NAT). However the risk of infection during window period (WP) might be missed by the immunoassay, which are responsible for most of the TTI. Zero window period might provide complete blood safety.

Aim: 1) To study sensitivity, specificity, positive predictive value (PPV), negative predictive value (NPV) and diagnostic accuracy (efficacy) of NAT over enzyme linked immuno sorbent assay (ELISA) methods in detecting TTI among blood donors

2) To assess the impact of introduction of individual donor - nucleic acid amplification test (ID-NAT) by all the blood banks and this study was conducted at blood bank, Gadag institute of medical sciences (GIMS), Gadag, Karnataka, India.

Methods: Over a period of 1year \& 6 months, a total number of 6000 blood donors, samples were subjected to ELISA and NAT.4th generation ELISA and NAT testing using, transcription mediated amplification(TMA) was used. The results were tabulated in an excel format and data was analyzed using Statistical Package for Social Sciences (SPSS) Software 21st version. Sensitivity, specificity, positive predictive value (PPV), negative predictive value(NPV) and diagnostic accuracy(efficacy) were calculated using Galen and Gambino method.

Result: A total of 6000 donors, blood samples were subjected to ELISA and NAT, among them 60 were seroreactive. 52 were NAT reactive and seronegative. The NAT yield was 1 in 113 . The present study showed $53.57 \%, 100 \%, 100 \%, 99.12 \%$ and $99.13 \%$ of sensitivity, specificity, PPV, NPV and diagnostic accuracy respectively.

Conclusion: NAT could detect human immunodeficiency virus (HIV), hepatitis B virus (HBV), and hepatitis C virus (HCV) cases in blood donor samples those that were undetected by immunoassays. Thus, NAT provides an additional layer of blood safety and provides safest blood pack for donation.
\end{abstract}

Keywords: Human immunodeficiency virus, Hepatitis B virus, Hepatitis C virus, Nucleic Acid Amplification Test, Enzyme Linked Immuno Sorbent Assay, Transfusion Transmissible Infections

\section{Introduction}

Blood safety in India is a challenging task and has become a top priority, with a population of 1.2 billion and a high prevalence rate of human immunodeficiency virus (HIV), hepatitis B virus (HBV) and hepatitis C virus (HCV). ${ }^{[1]}$

Laboratory screening of donated blood for infectious diseases is a key safety measure in preventing spread of serious diseases. The traditional method which is used for screening blood donations, known as immunoassay(or serology) testing. Immunoassays detect antibodies to viruses or viral antigens. With immunoassays; however, there is an interval between the donor's exposure to a virus until antibodies against the virus are produced, known as "window period"(WP). It is during this period there is risk of infection being missed in donated blood by immunoassay testing. These undetected window period infections are responsible for most of TTI. Thus, NAT takes care of the dynamics of WP of viruses and provide safe blood for donation. ${ }^{[2]}$

\section{Materials and Methods}

The present study is undertaken at blood bank, at Gadag Institute of Medical Sciences (GIMS), Gadag, over a period of 1 year \& 6 months from January 2016 to July 2017. All the blood donors (both voluntary and replacement) who qualified for blood donation were included in the study. 
The ethical clearance was obtained from the ethical clearance committee at GIMS, Gadag. After Physical examination medically fit donors were allowed to donate $350 \mathrm{ml}$ of blood. Samples were collected in pilot test tubes at the time of bleeding. All these samples were examined in the Transfusion Transmissible Disease screening laboratory at blood bank, GIMS, Gadag, using ELISA and NAT.

A total number of 6000 blood samples were subjected to screening for HIV, HCV\& HBV by using $4^{\text {th }}$ generation ELISA, Manufacturers guidelines provided by bio standards diagnostics were followed and all the blood samples were subjected to ID-NAT.

ID-NAT testing was done by using Transcription mediated amplification (TMA) which is simultaneous, single tube detection of HIV-1, HCV RNA and HBV DNA in donor's plasma.TMA process uses two enzymes-reverse transcriptase and RNA polymerase to produce hundreds of millions of copies of the targeted RNA sequences. This test takes place in a single tube involving steps of target capture, target amplification by TMA, detection of the amplification products with chemiluminescent probes by the hybridization protection assay.

The results were tabulated in an excel format and data was analyzed using SPSS Software $21^{\text {st }}$ version. Sensitivity, specificity, PPV, NPV and diagnostic accuracy were calculated using Galen and Gambino method as follows :

Sensitivity $=\frac{\boldsymbol{P}}{\boldsymbol{P}+\mathbb{N}} x 100$

Specificity $=\frac{\mathbb{N}}{\mathbb{N}+P} \times 100$

Positive $\mathbf{P}$ edictive Value $=\frac{\boldsymbol{P}}{\boldsymbol{P}+\boldsymbol{P}} \times 100$
Negative P edictive Value $=\frac{\mathbb{N}}{\mathbb{N}+\mathbb{N}} \times 100$

Efficacy $=\frac{\boldsymbol{P}+\mathbb{N}}{\boldsymbol{P}+P+\mathbb{P}+\mathbb{N}} x 100$

$\mathrm{TP}=$ True Positive $\quad \mathrm{FP}=$ False positive

$\mathrm{TN}=$ True Negative $\quad \mathrm{FN}=$ False Negative

These statistical values are interdependent statistical concepts indicating the accuracy of results.

\section{Result}

A total number of 6000 blood donations were screened in transfusion transmissible infections laboratory using ELISA and NAT. Among them majority of the donors 5760 (96\%) were male donors and remaining $240(4 \%)$ were female donors. Of these $1560(26 \%)$ were voluntary donors and $4440(74 \%)$ were replacement donors. First time donors were $5520(92 \%)$, whereas regular repeat donors were $480(8 \%)$.

Out of 6000 donors tested there were 60 sero-reactive cases, among them all 60 cases were HBV reactive. All the blood samples were tested by ID-NAT, of the 112 ID-NAT reactive samples, 52 were ID-NAT reactive, but seronegative. Out of 52, one was reactive for HIV-1, 1 for $\mathrm{HCV}$ and remaining 50 were reactive for HBV. There was no false positive case detected in the present study.

Nucleic Acid testing (NAT) yield: The combined yield (seronegative/NAT reactive) for HIV-1, HCV and HBV were 1 in 113.

On analysis of results, the present study showed sensitivity of $53.57 \%$, specificity of $100 \%$, PPV-100\%, NPV-99.12\% and diagnostic accuracy of $99.13 \%$.

Table 1:Interpretation of ELISA and NAT results .

\begin{tabular}{|c|c|c|c|}
\hline ELISA Results & NAT Results & & TOTAL \\
\hline POSITIVE & POSITIVE & TRUE POSITIVE & 60 \\
\hline NEGATIVE & NEGATIVE & TRUE NEGATIVE & 5888 \\
\hline NEGATIVE & POSITIVE & FALSE NEGATIVE & 52 \\
\hline POSITIVE & NEGATIVE & FALSE POSITIVE & 0 \\
\hline & & TOTAL & $\mathbf{6 0 0 0}$ \\
\hline
\end{tabular}


Table 2: comparison of Nat yield and Voluntary blood donation of present study with the other studies

\begin{tabular}{|c|c|c|}
\hline STUDIES & NAT YIELD & VOLUNTARY BLOOD DONATION (\%) \\
\hline Kumar R, Gupta S et al[1] & 1 in 753 & 35.2 \\
\hline Chatterjee $\mathrm{K}$, Coshic $\mathrm{P}$ et $\mathrm{al}^{[7]}$ & 1 in 2622 & \\
\hline Chaurasia R, Zaman S et al..$^{[11]}$ & 1 in 628 & 14.6 \\
\hline Agarwal N,Chatterjee $\mathrm{K}$ et a[ ${ }^{[12]}$ & 1 in 650 & \\
\hline Chandra T, Rizvi FN et al[13] & 1 in 156 & \\
\hline Jain $\mathrm{R}$, Aggarwal $\mathrm{P}$ et al ${ }^{[14]}$ & 1 in 2972 & 84.65 \\
\hline Makroo RN,Choudhary N et al[15] & 1 in 1528 & \\
\hline Present Study & 1 in 113 & 26 \\
\hline
\end{tabular}

\section{Discussion}

Blood safety is one of the major issues in India, because of the high prevalence of HIV, HCV and HBV with the relatively low percentage of volunteer donors and the lack of standardization of screening procedures among blood banks. The prevalence of HIV, HBV and HCV in India is respectively about $1 \%, 4 \%$ and $1.5 \%{ }^{[3]}$ Inspite of all the precautionary measures taken there is always a possibility to transmit the infection when blood from a recently infected donor fails to be identified by routine screening test. $^{[4]}$ This is because of window period, which puts blood recipients at a definite risk for transmissible diseases. With each unit of blood that is transfused there is $1 \%$ of chance of transmission of TTI. ${ }^{[5]}$

Since viremia precedes seroconversion by several days in case of HIV and several weeks for HBV and HCV, tests that detects viral nucleic acid are of prime importance in order to achieve the goal of zero risk for blood transfusion recipients. ${ }^{[6]}$

ID-NAT detects viremia earlier than current screening methods (immunoassays).It is highly sensitive and a direct test, which detects the viral ribonucleic acid (RNA) and deoxyribonucleic acid (DNA) by the amplification method. It reduces the window period by detecting low level of viral genomic material to the viruses. This further reduces the possibility of transmission via transfusion and also detects mutants and occult case. ${ }^{[7]}$

.The window period for detection by ID-NAT is 4.7 days for HIV-1, 2.2 days for $\mathrm{HCV}$ and 14.9 days for HBV. ${ }^{[8][9]}$ The corresponding window periods for serological tests are 15-20 days, 2-26 weeks, and 50-150 days respectively.

The benefits of ID-NAT are very important in patients who receive multiple blood transfusions for diseases such as thalassemia, hemophilia and cancers. Such patients need regular, repeated and life-long blood transfusions and are at higher risk of being infected with serious TTIs. In a survey by the National Thalassemia Welfare Society, among 551 multiple transfused patients with Thalassemia, 33 were HIV-positive, 89 were HCV-positive and 43 were HBV-positive. Transfusion associated common infections in thalassemic patients are Hepatitis C (2.2-44\%) followed by HBV (1.2-7.4\%) and HIV (0-9\%). ${ }^{[10]}$

ID-NAT testing was done by using Transcription mediated amplification (TMA). The advantages of using TMA are it allows for simultaneous testing of multiple viruses in a single test tube, thereby simplifying the assay process and it helps laboratories to reduce the steps for blood screening and shortens the processing time, thereby helping to get the results faster. ${ }^{[1]}$

In the present study majority of the donors were males $5760(96 \%)$ when compared to the females $240(4 \%)$ and majority of them donated blood for the first time $5520(92 \%)$ and remaining were $480(8 \%)$ were regular repeat donors. Similar observations were made by the study done by Kumar R, Gupta S et al. ${ }^{[1]}$ Majority of them were replacement donors $4440(74 \%)$ when compared to the voluntary donors $1560(26 \%)$. Similar observations were made by the study done by Chaurasia R, Zaman S et al. ${ }^{[11]}$

The NAT yield of 1 in 113 in our study assumes more significance when one considers the fact that single donation is used for generating 3 components that can be used by 3 recipients. Hence in effect the NAT yield becomes 3 times that is 3 in $113(2.65 \%)$ is indeed very significant. NAT yield and voluntary blood donation( $\%$ ) of various studies in comparison with the present study is shown in the table 2

This high yield of NAT in the present study is because of increased number of replacement donors when compared to voluntary donors and also due to the high prevalence of TTIs in India, further highlighting the need for NAT implementation in India.

On analysis of results of NAT and ELISA using SPSS software, the present study showed sensitivity of $53.57 \%$, 
specificity of $100 \%, \quad$ PPV- $100 \%, \quad$ NPV- $99.12 \%$ and diagnostic accuracy of $99.13 \%$, which is encouraging. Implementation of NAT would be more useful when compared to ELISA as window period infections are detected by NAT and it also prevents entry of infectious viruses into healthy recipients by means of blood transfusion.

\section{Conclusion}

Implementation of NAT had a significant effect on the safety of blood supply as it is a sensitive and specific technique which reduces the window period of detection of HIV, HBV and HCV. The need for NAT implementation depends on the prevalence and incidence rate of infections in blood donor population. NAT takes care of dynamics of window period of viruses and offers the safest blood pack for donation. The results in the present study support that the universal and routine use of ID-NAT for HIV, HBV and HCV by all blood banks would be an important step and have a remarkable effect in preventing TTI. NAT also serves as a valuable tool to achieve the motto of national blood safety organization of "vein to vein $100 \%$ safe blood"

\section{Reference}

1. Kumar R, Gupta S, Kaur A, Gupta M. Individual donor nucleic acid testing for human imunodefficiency virus-1, hepatitis $\mathrm{C}$ virus and hepatitis B virus and its role in blood safety. Asian J Transfus Sci. 2015;9:199-202.

2. Chigurupati P, Murthy KS. Automated nucleic acid amplification testing in blood banks: an additional layer of blood safety. Asian J Transfus Sci. 2015;9:9-11.

3. Dodd RY, Notari EP 4 th, Stramer SL. Current prevalence and incidence of infectious disease markers and estimated window-period risk in the American Red Cross blood donor population. Transfusion 2002;42:975-9.

4. Kapoor D, Saxena R, Sood B, Sarin SK. Blood transfusion practices in India: Results of a national survey. Indian $\mathrm{J}$ Gastroenterol 2000;19:64-7.

5. Garg S, Mathur DR, Garg DK. Comparison of seropositivity of HIV, HBV, HCV and syphilis in replacement and voluntary blood donors in western India. Indian J Pathol Microbiol.2001;44:409-12.

6. Busch MP, Kleinman SH, Jackson B, Stramer SL, Hewlett I, Preston S. Committee report. Nucleic acid amplification testing of blood donors for transfusion-transmitted infectious diseases: Report of the Interorganizational Task Force on Nucleic Acid Amplification Testing of Blood Donors. Transfusion. 2000;40:143-59.

7. Chatterjee K, Coshic P, Borgohain M, Premchand, Thapliyal RM, Chakroborty S, et al. Individual donor nucleic acid testing for blood safety against HIV-1 and hepatitis B and $\mathrm{C}$ viruses in a tertiary care hospital. Natl Med J India. 2012;25:207-9.

8. Palla P, Vatteroni ML, Vacri L, Maggi F, Baicchi U. HIV-1 NAT minipool during the pre-seroconversion window period: Detection of a repeat blood donor. Vox Sang.2006;90:59-62.

9. Weusten J, Vermeulen M, van Drimmelen H, Lelie N. Refinement of a viral transmission risk model for blood donations in seroconversion window phase screened by nucleic acid testing in different pool sizes and repeat test algorithms. Transfusion. 2011;51:203-15.

10. Jain R, Perkins J, Johnson ST, Desai P, Khatri A, Chudgar U, et al. A prospective study for prevalence and/or development of transfusion-transmitted infections in multiply transfused thalassemia major patients. Asian J Transfus Sci. 2012;6:151-4.

11. Chaurasia R, Zaman S, Das B, Chatterjee K. Screening donated blood for transfusion transmitted infections by serology along with NAT and response rate to notification of reactive results: An Indian experience. J Blood Transfus. 2014;2014:412105.

12. Agarwal N, Chatterjee K, Coshic P, Borgohain M. Nucleic acid testing for blood banks: An experience from a tertiary care centre in New Delhi, India. Transfus Apher Sci 2013;49:482-4.

13. Chandra S T. Rizvi F N, Agarwal D . Nucleic acid testing in blood donors of northern india: a single centre experience. International Journal of Contemporary Medical Research 2016;3(6):1818-1821.

14. Jain R, Aggarwal P, Gupta GN. Need for nucleic acid testing in countries with high prevalence of transfusion-transmitted infections. ISRN Hematol 2012. 2012718671.

15. Makroo RN, Choudhury N, Jagannathan L, PariharMalhotra M, Raina V, Chaudhary RK, et al. Multicenter evaluation of individual donor nucleic acid testing (NAT) for simultaneous detection of human immunodeficiency virus -1 \&amp; hepatitis B \&amp; C viruses in Indian blood donors. Indian J Med Res. 2008;127:140-7.

*Corresponding author:

Dr. Basavaraj P Bommanahalli, Professor and Head, Department of Pathology, Gadag Institute Of Medical sciences, Gadag-582103, Karnataka, India. Phone: +91 9916088221

Email: basupath@rediffmail.com

Financial or other Competing Interests: None. 